\title{
TEMAS TRANSVERSALES, CIUDADANÍA Y EDUCACIÓN EN VALORES: DE LA LOGSE (1990) A LA LOMLOE (2020)
}

\section{CROSS-CURRICULAR TEACHING, CITIZENSHIP AND VALUES EDUCATION: FROM LOGSE (1990) TO LOMLOE (2020)}

https://doi.org/10.15304/ie.30.7092

\author{
Raquel Vázquez Ramil \\ Universidad de Valladolid \\ mariaraquel.vazquez@uva.es \\ Ángel Serafín Porto Ucha \\ Universidad de Santiago de Compostela \\ angelserafin.porto@usc.es
}

\begin{abstract}
La educación procura formar el carácter, hacer del sujeto una persona con cualidades esenciales generales, de las que no podrá prescindir nunca y necesitará siempre si ha de ser como debe.
\end{abstract}

Concepción Arenal (1892) ${ }^{1}$.

\section{RESUMEN}

En estos momentos de intensa inquietud social debido a la incidencia de coronavirus en todo el mundo y, de forma trágica, en España, hacemos una revisión de las principales normas educativas de los últimos treinta años, cada vez más frecuentes al hilo de los cambios políticos, y cada vez también más polémicas, como reflejo de una sociedad sometida a profundas alteraciones. Para dar respuesta a esas demandas, la educación obligatoria no aspira a transmitir solo conocimientos, sino que busca asentar actitudes, desarrollar principios y contribuir a la plenitud de la persona a través de los temas transversales, en ocasiones, o de áreas o materias de carácter globalizador, como Educación para la Ciudadanía o Educación en Valores Cívicos y Éticos. ¿En qué punto estamos? En el umbral de un nuevo cambio legislativo que debe apresurarse para dotar de valores a los ciudadanos y ciudadanas del futuro.

Palabras clave: Temas transversales; Educación para la Ciudadanía; Educación en Valores; Cultura del esfuerzo; Legislación educativa.

Recibido: 8/IX/2020. Aceptado: IX/2020

1 «La educación de la mujer», informe presentado por Concepción Arenal en el Congreso Pedagógico Nacional de 1892 (sección 5 $5^{\mathrm{a}}$ ). 


\begin{abstract}
At this time of intense social concern due to the incidence of coronavirus worldwide and, tragically, in Spain, we are reviewing the main educational pieces of legislation of the last thirty years, increasingly frequent in line with political changes, also more and more controversial, reflecting a society that undergoes deep alterations. To satisfy these demands, compulsory education doesn't want to transmit only knowledge, but it seeks to settle attitudes, develop principles and contribute to the person's complete fulfilment through cross-curricular teaching, sometimes, or by means of areas or subjects of globalizing nature, such as Education for Citizenship or Civic and Ethical Values Education. Where are we now? On the threshold of a new legislative change that must be very quick to provide true values to the future citizens.
\end{abstract}

Key words: Cross-curricular teaching; Education for Citizenship; Values Education; Culture of hard work; Educational Laws.

\title{
1. LOS TEMAS TRANSVERSALES EN LA LEGISLACIÓN EDUCATIVA: LA LOGSE Y SUS PRECEDENTES
}

Los temas transversales son contenidos que por su carácter no encajan en un área o asignatura, sino que se abordan en varias o en todas, como indica Rosales (2015: 144). Se refieren a problemas del momento y a la realidad social (Bolívar, 1995) y persiguen la transmisión de actitudes y valores morales cívicos (Esteban Moreno, 2003: 100). González Lucini (1993) identificó transversalidad con valores, marcando una línea teórica que tendrá largo arraigo, pero que aún hoy sigue buscando concreción en la legislación educativa, como lo demuestran los frecuentes cambios en la misma desde principios de los años 90 hasta el actual Proyecto de Ley Orgánica de modificación de la LOE (LOMLOE) ${ }^{2}$.

La Constitución Española de 1978 dedica el artículo 27 a la educación, y el punto 2 establece que «la educación tendrá por objeto el pleno desarrollo de la personalidad humana en el respeto a los principios democráticos de convivencia y a los derechos y libertades fundamentales». Pero ya antes, desde la Ley General de Educación de 1970, se aprecia el interés por generalizar la educación en respuesta a las necesidades de una sociedad que había cambiado más rápido que el régimen político que la gobernaba. Prueba de ello es la Orden ministerial de 29 de noviembre de $1976^{3}$, que establecía nuevos contenidos en el área social de la Educación General Básica (EGB); se habla entonces de educación para la convivencia con el fin de construir una nueva sociedad en la que la persona sea sujeto de derechos y deberes; para ello se incluyen, en principio con carácter experimental, contenidos de carácter cívico-social en la se-

2 Aprobado en el Consejo de Ministros del 15 de febrero de 2019 y publicado en el Boletín Oficial de las Cortes el 22 de febrero de 2019; en la actualidad (septiembre de 2020) se encuentra en trámite parlamentario, tras la forzosa interrupción de la actividad del Congreso por el estado de alarma derivado de la pandemia de coronavirus.

3 Derogatoria de la Orden de 25 de junio de 1974, que establecía nuevos contenidos en las orientaciones pedagógicas del área social en la Segunda Etapa de Educación General Básica, y cuyo carácter era muy distinto. 
gunda etapa de EGB, y que son: la convivencia y su alcance, la democracia y sus fundamentos, el conocimiento de uno mismo y la autorrealización personal, el patrimonio histórico-artístico y su defensa, y la conciencia mundial. No se habla aún de transversalidad, pero sí de contenidos que formen a la persona y al ciudadano para la convivencia y el progreso.

La LOGSE (Ley Orgánica 1/1990, de 3 de abril, de Ordenación General del Sistema Educativo) tampoco cita expresamente los temas transversales, pero supone un cambio de perspectiva notable con respecto a la legislación anterior, al declarar, en su artículo 1, que el sistema educativo español atenderá al «pleno desarrollo de la personalidad del alumno», teniendo como principio básico la educación permanente. El artículo 2.3 indica además que la actividad educativa se cimenta en una serie de criterios, el primero de los cuales es «la formación personalizada, que propicie una educación integral en conocimientos, destrezas y valores morales de los alumnos en todos los ámbitos de la vida, personal, familiar, social y profesional».

El Real Decreto 1344/1991, que concreta el currículum de Educación Primaria, era más explícito al declarar que la educación integral no se conseguía con acumulación de conceptos y contenidos académicos, sino que había que contemplar aspectos esenciales para el desarrollo de las personas, a saber, «habilidades prácticas, actitudes y valores», siendo la educación social y la educación moral elementos básicos del proceso educativo ¿Y cómo se alcanzaba un propósito tan ambicioso? Incluyendo elementos formativos en consonancia con la sociedad del momento: educación para la paz, la salud, la igualdad entre los sexos, educación ambiental, sexual, del consumidor y vial.

Para facilitar la puesta en práctica de estos presupuestos, el Ministerio de Educación elabora un conjunto de Materiales para la reforma, recogidos en las conocidas como «cajas rojas». En Educación Infantil se contemplan los temas transversales como los que «engloban múltiples contenidos que difícilmente se pueden adscribir a ninguna de las áreas» (Pablo López, 1992: 86), pero que en un modelo que aspira a la formación integral de la persona han de estar presentes. Son: la educación moral y para la paz, la educación para la igualdad de oportunidades de ambos sexos, la educación para la salud, la educación vial, la educación ambiental y la educación del consumidor.

El volumen dedicado a Educación Primaria es más explícito y aclara las pretensiones y alcance de los temas transversales en esa etapa; son (Zenón Gómez, 1992: 319-323): implantar un modelo de persona desde una concepción humanista; el desarrollo integral del individuo; la apuesta por la educación en valores; la relación de la escuela con el entorno, y la incorporación de nuevas enseñanzas. El paradigma en torno al cual se articulan todos los temas transversales es la educación moral y cívica, que en su primer aspecto forma a la persona individual y en el segundo a la que convive con otros. En este punto es interesante la atención que se dedica al peso de currículum oculto en la transmisión de valores dentro de la institución escolar y a la necesidad de hacerlo explícito para corregir comportamientos no deseados.

El completo documento ofrece una serie de estrategias para la educación en valores: la discusión de dilemas morales, que permite el desarrollo de la capacidad de razonamiento en 
situaciones problemáticas; la comprensión crítica; y la clarificación de valores, que se acompañan de materiales y ejercicios útiles.

El volumen dedicado a Educación Secundaria Obligatoria subraya la importancia de los temas transversales y ofrece recomendaciones sobre su tratamiento en los distintos proyectos curriculares, aunque se apunta, muy acertadamente, al peligro de que no se desarrollen por su propio carácter integrador, que puede llegar a ser disgregador, como bien indica Rosales al decir que los temas transversales, por su índole multidisciplinar, exigen una imprescindible coordinación entre todo el profesorado «si se quiere lograr una intervención coherente y no fragmentada, si se quiere dar el paso cualitativo tan necesario de la multidisciplinariedad a la interdisciplinaridad» (2019: 115).

Los temas transversales propuestos en la LOGSE tienen un claro significado social y se orientan, fundamentalmente, a la construcción de la ciudadanía, pero algunos autores apreciaron en ellos la tendencia a no considerar «los grandes valores antropológicos y espirituales» (Parra Ortiz, 2003: 74). El debate sobre la jerarquía de valores en educación tiene un largo recorrido y brotará con cierta crispación en el siguiente cambio normativo, el dictado por la LOE.

\section{DE LA CULTURA DEL ESFUERZO A LA EDUCACIÓN PARA LA CIUDADANÍA, LA LOE Y SUS ECOS}

Tras el asentamiento de la democracia, desde la última década del siglo XX, la educación se va a convertir en tema de debate político de primer orden, hasta el punto de que sucesivos gobiernos proponen y, algunas veces, consiguen implantar su propia legislación educativa en períodos cada vez más breves y al hilo de circunstancias que, en la actualidad, son tan apremiantes como una situación de pandemia mundial. En el caso de la LOGSE y su puesta en práctica, la crisis económica, el inmovilismo del profesorado y la «abulia de la clase política», contribuyeron, según López Serrano (2019: 568) a su defectuoso funcionamiento y a suscitar clamores de reforma desde todos los ámbitos.

El segundo gobierno del PP, presidido por José $\mathrm{M}^{\mathrm{a}}$ Aznar y con Pilar del Castillo como ministra de Educación, elaboró una ley educativa de carácter reformista que no llegó a entrar en vigor, pero que marca distancias con respecto a la LOGSE e ilustra lo que serán los tensos debates sobre el modelo educativo en la España del siglo XXI, en los cuales, como no podía ser menos, tienen papel esencial los temas transversales (incluso cuando no se citan) y los «valores».

La Ley Orgánica 10/2002 de Calidad de la Educación (LOCE) indica en su exposición de motivos que, como su nombre señala, el fin de la norma es garantizar una «educación de calidad» para todos, recurriendo a «los valores humanistas propios de nuestra tradición cultural europea». Se establecía un objetivo eminentemente práctico, el de reducir las altas tasas de fracaso escolar, sobre todo en la educación secundaria; y se destacaba un valor no mencionado en la ley anterior, el de la «cultura del esfuerzo» como garantía de progreso personal, «porque sin esfuerzo no hay aprendizaje». Precisamente es el esfuerzo el llamado a corregir las diferen- 
cias sociales, de ahí la importancia que se da a las pruebas de evaluación a diferentes niveles. La LOGSE también había aspirado a mejorar la calidad de la enseñanza, pero sin utilizar el concepto esfuerzo, sino, como expresa en su preámbulo, modernizando los centros, mejorando la consideración social de los docentes, y con la participación activa de todos los miembros de la comunidad educativa.

Ciertos sectores tildaron de neoliberal ese énfasis. en el esfuerzo y advirtieron de que contribuía a la competitividad y a la segregación del alumnado, justificaba el discurso del mérito, la concepción clasista de la educación, la estratificación y control del alumnado y la no atención a la diversidad (Gil Rivero 2003: 1); otros, considerando el esfuerzo como paradigma actitudinal, afirmaron que «si la sociedad no se apropia del esfuerzo, no habrá ley que pueda imponerlo» (Vinuesa Angulo 2002: 213) y subrayaron la confrontación que se imponía entre el aprendizaje lúdico como cultura del mínimo esfuerzo, implícito en la norma legal precedente, y la laboriosidad como cultura del esfuerzo, defendida en la LOCE. La perspectiva intermedia o ponderada es la defendida por autores como Gervilla Castillo, cuando afirma que «Ser persona educada no es un regalo, sino una conquista, una carrera (“currículum") en la que es imposible, sin sudor, alcanzar la meta: la formación personal y la construcción social» (2003: 111), a la cual se llega mediante la argumentación, la reflexión y la crítica.

Tras las elecciones generales de marzo de 2004, que dieron la victoria al PSOE, se suspendió la entrada en vigor de la $\mathrm{LOCE}^{4}$, estableciéndose un nuevo calendario de aplicación que nunca llegará a ser efectivo, pues se procedió a una nueva reforma de la legislación educativa.

La Ley Orgánica 2/2006, de 3 de mayo, de Educación (LOE), establece en el preámbulo que la educación es la vía para construir la personalidad y desarrollar las capacidades de las personas, conformar la identidad y la comprensión de la realidad, además de actuar como «el medio más adecuado para garantizar el ejercicio de la ciudadanía democrática». Recalca la importancia del esfuerzo para conseguir la calidad y la equidad educativas, pero del esfuerzo compartido, no solo del estudiante, sino también de las «familias, el profesorado, los centros docentes, las administraciones educativas y, en última instancia, de la sociedad en su conjunto, responsable última de la calidad del sistema educativo».

En lo que atañe al currículo, la LOE no alude a elementos transversales de forma explícita, pero introduce la educación para la ciudadanía en lugar destacado como materia con distintas denominaciones en los diferentes niveles de escolarización obligatoria, con el fin de (BOE, $\left.\mathrm{n}^{\mathrm{o}} 106,2006: 17163\right)$ :

Ofrecer a todos los estudiantes un espacio de reflexión, análisis y estudio acerca de las características fundamentales y el funcionamiento de un régimen democrático, de los principios y derechos establecidos en la Constitución española y en los tratados y las declaraciones universales de los derechos humanos, así como de los valores comunes que constituyen el sustrato de la ciudadanía democrática en un contexto global.

4 Real Decreto 1318/2004, de 28 de mayo, por el que se modifica el Real Decreto 827/2003, de 27 de junio, por el que se establece el calendario de aplicación de la nueva ordenación del sistema educativo, establecida por la Ley Orgánica 10/2002, de 23 de diciembre, de Calidad de la Educación. 
La LOE entró en vigor en el curso 2006-2007. El Real Decreto 1513/2006, que regulaba las enseñanzas mínimas en la etapa de Educación Primaria, establecía cinco áreas: Conocimiento del medio natural, social y cultural y Educación artística; Educación física; Lengua castellana y literatura y, si la hubiere, lengua cooficial y literatura; Lengua extranjera; y Matemáticas. El punto 2 del artículo 4, añadía que «en uno de los cursos del tercer ciclo de la etapa se añadirá el área de Educación para la ciudadanía y los derechos humanos, en la que se prestará especial atención a la igualdad entre hombres y mujeres». Los contenidos de la nueva área se organizaban en tres bloques: el individuo y las relaciones interpersonales y sociales; la vida en comunidad; y vivir en sociedad.

El Real Decreto 1631/2006, que establecía las enseñanzas mínimas en Educación Secundaria, introduce la Educación para la ciudadanía y los derechos humanos en uno de los tres primeros cursos, prestando de nuevo especial atención a la igualdad entre los sexos; en cuarto curso es obligatoria la materia Educación ético-cívica.

La LOE supuso un cambio sustancial con respecto a la legislación anterior; cambio que quedó difuminado por el intensísimo debate político y social en torno a la materia de educación para la ciudadanía, considerada desde algunos sectores como partidista y adoctrinadora, y muy protestada en todo tipo de tribunas e incluso en concurridas manifestaciones públicas. El debate sobre la educación y, más en concreto, sobre un valor de la educación, el de formar ciudadanos, se convirtió así en noticia de primera plana y materia de opinión popular en una corriente cuyos ecos aún se escuchan hoy. Ha habido incluso un movimiento de «objeción de conciencia» (Martín Sánchez, 2009) de padres que no deseaban que sus hijos recibiesen las enseñanzas de dicha asignatura y que llegó a los tribunales, dando lugar a una amplia y compleja jurisprudencia (Gómez Orfanel 2009).

En la inclusión de la educación para la ciudadanía, si nos ceñimos a consideraciones estrictamente pedagógicas, existía la pretensión de suplir las deficiencias de las transversalidad propuesta por la LOGSE, que nunca llegó a ser efectiva debido al peso de los contenidos disciplinares, sobre todo en Educación Secundaria, como apunta Rodríguez Lestegás (2007: 85). Bolívar (2007) acertadamente señaló la importancia de la enseñanza de educación para la ciudadanía, pero sin restringirla a un área, en Primaria, o a una materia, en Secundaria, sino recuperando la acción transversal para que calase en todo lo que se transmitía en el centro escolar y aún fuera de él, pues la esencia ciudadana no es un contenido.

Sin embargo, la realidad ha sido más tozuda en este aspecto, convirtiendo una oportunidad en polémica, tal vez por el difuso recuerdo de la rígida formación política de otra época, por un lado, y por el temor a la interferencia del Estado en terrenos considerados estrictamente familiares, lo cual nos lleva al espinoso enfrentamiento familia-Estado con la escuela en medio y nos remite a las palabras de Bolívar (2007: 59),

Asumir aisladamente la tarea educativa, ante la falta de vínculos de articulación entre familia, escuela y medios de comunicación, es una fuente de tensiones y desmoralización docente. De ahí la necesidad de actuar paralelamente en estos otros campos, para no hacer recaer en la escuela responsabilidades que también están fuera. 
Los intensos debates condujeron a la modificación del contenido y alcance de la educación para la ciudadanía por Real Decreto 1190/2012, poco antes de la aprobación de una nueva Ley Orgánica de Educación, con el Partido Popular en el gobierno; el cambio era, en principio, de matiz, y pretendía que la tarea de educación en democracia se hiciese «de un modo compartido y no excluyente entre la familia, las instituciones públicas, los centros educativos y la misma sociedad», ya que «el Estado tiene la obligación de asegurar que los ciudadanos conozcan la Constitución, el funcionamiento de las instituciones públicas y cuanto se refiere a los derechos y libertades».

En realidad, la educación para la ciudadanía venía a ser la culminación de intentos anteriores de incorporar los valores cívicos al currículum, como señala Gómez Rodríguez (2008); la LOGSE contemplaba su transmisión transversal, precisamente, en las diferentes materias. Pero se convirtió en «algo más que una asignatura» (Bolívar, 2008) y generó una enconada polémica entre fe religiosa y libertad cívica, pugna que España ya había vivido en épocas anteriores, y que como bien analizaba Fernández Soria (2008) presentaba difícil solución.

\section{LA LOMCE: A LA BÚSQUEDA DE LA CALIDAD EDUCATIVA}

En las elecciones generales de noviembre de 2011 obtuvo mayoría absoluta el Partido Popular, con Mariano Rajoy como presidente, haciéndose cargo de la cartera de Educación José Ignacio Wert, impulsor y cabeza visible de una nueva reforma educativa, la Ley Orgánica 8/2013 para la Mejora de la Calidad Educativa (LOMCE), también conocida como Ley Wert ${ }^{5}$. Una de las primeras medidas del ministro fue anunciar la sustitución de la controvertida Educación para la Ciudadanía por la materia Educación Cívica y Constitucional; naturalmente, el anuncio complació a determinados sectores, pero enervó a otros, creando un clima convulso que convirtió la educación en caballo de batalla, con frecuentes huelgas de estudiantes y profesores, y niveles de tensión inéditos en torno al cariz ideológico de los contenidos educativos y a los recortes económicos.

En medio de todo ello se aprueba la LOMCE en diciembre de 2013. La nueva norma define la educación como «el motor que promueve el bienestar de un país», un símil claramente industrial, y dicta que en su transformación ha de participar toda la sociedad (empresas, asociaciones, sindicatos) y de manera muy particular las familias, que son «las primeras responsables de la educación de sus hijos y por ello el sistema educativo tiene que contar con la familia y confiar en sus decisiones». Se marca así una línea clara que da protagonismo a las familias en la educación de los hijos, frente al papel del Estado defendido, por ejemplo, en la LOE, y de nuevo en la actualidad.

En España contadas leyes educativas se conocen por el nombre de los ministros del ramo, es el caso de la famosa Ley Moyano de 1857 y la Ley Wert o LOMCE. En la actualidad, se habla de la Ley Celaá, en referencia a la LOMLOE impulsada por la ministra socialista Isabel Celaá Diéguez. 
La reforma perseguía reducir las tasas de abandono temprano, alcanzar buenos resultados de acuerdo con criterios internacionales, «mejorar la empleabilidad y estimular el espíritu emprendedor de los estudiantes», principios esencialmente prácticos y económicos que convenían al momento de crisis que estaba viviendo el país, pero que difícilmente podían alcanzarse con medidas colaterales de recortes en educación. Desde amplios sectores se tachó a la nueva Ley de imponer una «concepción economicista y mercantilista de la sociedad» (Bayona Aznar 2013: 13), de fomentar la competitividad frente a la equidad a través de pruebas de evaluación que remitían a las antiguas reválidas, y de eliminar las «materias incómodas», en referencia a la Educación para la Ciudadanía, la Ética o las Ciencias del Mundo Contemporáneo.

La agitación social que en su momento provocó la LOE subió de tono con la LOMCE y se mantiene hoy, como veremos, y el motivo es la introducción de un modelo educativo que, como todos los modelos educativos, se nutre de valores a veces enfrentados. Las críticas a la LOMCE han sido tan numerosas que es imposible compendiarlas, pero denotan un preocupante distanciamiento entre la norma y la sociedad y, en última instancia, el escaso calado que han tenido los elementos transversales en la educación obligatoria. Se discute el modelo de persona, premisa básica sin la cual no puede haber acuerdo en nada; como indica Subirats Martòri (2014: 54): «El homo economicus sustituye al ciudadano y la ciudadana desaparece». En cuanto a la Educación para la Ciudadanía, la LOMCE se remitía a la Recomendación del Parlamento Europeo y del Consejo de 18 de diciembre de 2006 sobre las competencias clave para el aprendizaje permanente, abordando la cuestión «de forma transversal al incorporar la educación cívica y constitucional a todas las asignaturas durante la educación básica, de forma que la adquisición de competencias sociales y cívicas se incluya en la dinámica cotidiana de los procesos de enseñanza y aprendizaje». En Educación Primaria se contemplaba la asignatura específica Valores sociales y cívicos, alternativa de la Religión a elección de los padres o tutores; y en el primer ciclo de Educación Secundaria Obligatoria Valores éticos, igualmente como alternativa a la materia específica de Religión.

El Real Decreto 126/2014 que establece el currículo básico de Educación Primaria dispone en el artículo 10.1:

Sin perjuicio de su tratamiento específico en algunas de las asignaturas de cada etapa, la comprensión lectora, la expresión oral y escrita, la comunicación audiovisual, las Tecnologías de la Información y la Comunicación, el emprendimiento y la educación cívica y constitucional se trabajarán en todas las asignaturas.

Las administraciones educativas deben incorporar, transversalmente, elementos curriculares relacionados con los siguientes ámbitos de la Educación Primaria:

Inclusión de las personas con discapacidad y atención a la diversidad.

Igualdad efectiva entre hombres y mujeres y prevención de la violencia de género.

Prevención y resolución pacífica de conflictos en todos los ámbitos.

Desarrollo sostenible y medio ambiente.

Riesgos de utilización de las Tecnologías de la Información y Comunicación. 
Protección ante emergencias y catástrofes.

Desarrollo y afianzamiento del espíritu emprendedor.

Atención a la actividad física y a una dieta equilibrada.

Fomento de la seguridad vial y prevención de los accidentes de tráfico.

El Real Decreto 1105/2014, que establece el currículo de la Educación Secundaria Obligatoria y el Bachillerato, contempla los elementos transversales en el artículo 6, declarando en el punto 1:

En Educación Secundaria Obligatoria, sin perjuicio de su tratamiento específico en algunas de las materias de cada etapa, la comprensión lectora, la expresión oral y escrita, la comunicación audiovisual, las Tecnologías de la Información y la Comunicación, el emprendimiento y la educación cívica y constitucional se trabajarán en todas las materias.

En cuanto al desarrollo curricular de dichos elementos transversales por parte de las administraciones educativas, se recomienda:

La igualdad efectiva entre hombres y mujeres y la prevención de la violencia de género y contra la discapacidad.

Desarrollo y afianzamiento del espíritu emprendedor y fomento de la ética empresarial.

Atención a la actividad física y a la dieta equilibrada.

Mejora de la convivencia vial y prevención de los accidentes de tráfico.

El énfasis que la LOMCE pone en los elementos transversales no oculta las dificultades de su puesta en práctica, como hemos visto desde el inicio, no solo para el profesorado y dentro de las aulas, puesto que, como indica Rosales (2015: 155)

La educación en temas transversales, como cuestiones de relevante interés social y personal, se vincula con todos aquellos contextos o escenarios en los que se desarrolla la vida de la persona, y en este sentido podríamos recordar cuáles son los tres grandes agentes educativos, tradicionalmente identificados como la familia, la escuela y la comunidad.

El Informe Eurydice definía la educación para la ciudadanía como la materia que en los centros escolares debe «fomentar la convivencia armoniosa y el desarrollo mutuo de las personas y de las comunidades de las que forman parte» (Comisión Europea, 2017: 8), con el fin de que los alumnos se conviertan en ciudadanos activos, informados y conscientes, capaces de responsabilizarse de sí mismos y de la comunidad en la que viven, algo sumamente necesario, sobre todo en la actualidad. El Informe engloba en el concepto educación para la ciudadanía, la educación en valores cívicos y éticos, y denominaciones similares que existen en otros países de la Unión Europea; es un estudio esclarecedor, por la cantidad de datos que aporta y por el tratamiento de los mismos, y en el caso de España tiene en cuenta las peculiaridades de algunas comunidades autónomas, caso de Cataluña, Andalucía o Galicia. Entre otros aspectos, incide en que el éxito de los conocimientos de la educación para la ciudadanía en todas partes depende del conocimiento de los profesores, de su preparación metodológica, sus competencias sociales, y la capacidad de reflexión, evaluación y mejora (142-144). 


\section{OTRA VUELTA DE TUERCA, LA LOMLOE Y LA REFORMA DE LA REFORMA EDUCATIVA}

Tras las elecciones generales de abril y noviembre de 2019, el gobierno de coalición PSOE-UP presidido por Pedro Sánchez, con la ministra Isabel Celaá en la cartera de Educación, ha elaborado un Proyecto de Ley Orgánica que modifica la Ley Orgánica 2/2006, de Educación, conocido como LOMLOE, aprobado en la sesión del Consejo de Ministros de 3 de marzo de 2020 y pendiente de culminación de trámites parlamentarios debido a la suspensión de la actividad política por mor de la pandemia de coronavirus. En la declaración de motivos, el Proyecto de Ley se hace eco de las numerosas críticas vertidas contra la LOMCE y se encamina a corregir aspectos «indeseados», recuperando el espíritu de la LOE con las modificaciones que impone el paso del tiempo y la adaptación a nuevas exigencias.

La transversalidad en Educación Primaria se contempla atendiendo en todas las áreas a la comprensión lectora, la expresión oral y escrita, la comunicación audiovisual, las tecnologías de la información y la comunicación, el fomento de la creatividad y del espíritu científico, la educación para la salud, incluida la afectivo-sexual y la educación emocional, dedicando un tiempo diario a la lectura. Además, en el tercer ciclo de Educación Primaria se contempla la inclusión de la materia Valores cívicos y éticos, y se oferta obligatoriamente la de Religión «sin asignatura espejo»; el planteamiento en estos puntos es similar en la ESO, reforzando además la orientación con perspectiva de género. La pretendida entrada en vigor de la nueva Ley en el curso 2020-2021 se ha visto comprometida por el estado de alarma y por la oposición de los grupos parlamentarios del Partido Popular, Ciudadanos y Vox, que han presentado enmiendas a la totalidad.

Nos encontramos en un momento crítico, con la incorporación de alumnos y alumnas a las aulas en precario por el temor a la expansión del coronavirus en espacios en los que, forzosamente, conviven durante largo tiempo niños y adultos. La sociedad está mucho más sensibilizada con el problema sanitario, que es apremiante, pero a él se añade la falta de consistencia de las normas genéricas que regulan la educación obligatoria en nuestro país.

Cuando se presenta una situación tan grave como una pandemia, que afecta a todos y todas, ¿qué ha hecho la escuela para prepararnos? ¿Qué hemos aprendido transversalmente o en línea recta? Y sobre todo, ¿cómo vamos a preparar la escuela a partir de ahora? ¿A qué valores apelamos?

El $B O E$ no tiene todas las respuestas porque es la sociedad entera la que debe comprometerse en educar para vivir, para que todos y todas tengamos las cualidades que definen a la persona como tal y que nos permiten ser como debemos ser, reiterando las palabras de Concepción Arenal hace ciento treinta años. 


\section{REFERENCIAS BIBLIOGRÁFICAS}

Arenal, C. (1974). La emancipación de la mujer en España. Madrid: Júcar.

Bayona Aznar, B. (2013). Los ejes de la LOMCE. Forum Aragón: revista digital de FEAEAragón sobre organización y gestión educativa, (7), 13-15.

Bolívar Botía, A. (1995). La educación en valores y actitudes. Madrid: Anaya/Alauda.

Bolívar Botía, A. (2007). La educación para la Ciudadanía: su lugar en el currículum. Idea la Mancha: Revista de Educación de Castilla-La Mancha, (4), 55-59.

Bolívar Botía, A. (2008). Educación para la ciudadanía. Algo más que una asignatura. Barcelona: Graò.

Comisión Europea/EACEA/Eurydice (2017). La educación para la ciudadanía en el contexto escolar europeo - 2017. Informe de Eurydice. Luxemburgo: Oficina de Publicaciones de la Unión Europea. DOI: https://doi.org/10.2797/90329

Esteban Moreno, R. M. (2003). Educación en valores. Programa para su desarrollo en la Educación Secundaria Obligatoria. Tendencias pedagógicas, (8), 99-106.

Fernández Soria, J. M. (2008). Educación para la Ciudadanía y los Derechos Humanos: controversias en torno a una asignatura (o entre ética pública y ética privada). Trasatlántica de Educación, (4), 45-64.

Gervilla Castillo, E. (2003). Pedagogía del esfuerzo y cultura del placer. Revista española de pedagogía, 61(224), 97-114. Disponible en: https://www.jstor.org/stable/23764439

Gil Rivero, J. (2003). Un análisis crítico de la Ley Orgánica de Calidad de la Educación. Laberinto, (11), 1-14.

Gómez Orfanel, G. (2009). Jurisprudencia española sobre educación para la ciudadanía. Anuario de Derechos Humanos. Nueva Época, 10, 261-283.

Gómez Rodríguez, A. E. (2008) Educación para la ciudadanía: una aproximación al estado de la cuestión. Enseñanza de las ciencias sociales: revista de investigación, (7), 131-140.

González Lucini, F. (1993). Temas transversales y educación en valores. Madrid: Alauda.

López Serrano, M. J. (2019). 40 años de Leyes y didácticas educativas. Intervencionismo político en la educación española. Anuario Jurídico y Económico Escurialense, LII, 559-572.

Martín Sánchez, I. (2009). Objeción de conciencia y educación para la ciudadanía. Revista Jurídica de la Universidad Autónoma de Madrid ( RJUAM), (19), 211-228.

Pablo López, P. de (Coord.) (1992). Materiales para la reforma. Educación Infantil. Madrid: Centro de Publicaciones del MEC.

Parra Ortiz, J. M. (2003). La educación en valores y su práctica en el aula. Tendencia pedagógicas, 8, 69-88.

Rodríguez Lestegás, F. (2007). Educación para la ciudadanía: el gato ya tiene su cascabel. Contextos educativos. Revista de Educación, (10), 81-90, DOI: https://doi.org/10.18172/ con.580

Rosales López, C. (2015). Evolución y desarrollo actual de los temas transversales: posibilidades y límites. Foro de Educación, 13(18), 143-160. DOI: https://doi.org/10.14516/ fde.2015.013.018.008 
Rosales López, C. (2019). ¿Cuáles son los ámbitos de transversalidad educativa? Innovación Educativa, (29), 109-123. DOI: https://doi.org/10.15304/ie.29.6023

Subirats Martòri, M. (2014). La LOMCE: hacia una educación antidemocrática. Revista interuniversitaria de formación del profesorado, (81), 45-58.

Vinuesa Angulo, J. M. (2002) La cultura del esfuerzo. Revista de Educación, (329), 207-218.

Zenón Gómez. J. (1992). Materiales para la reforma. Educación Primaria (segundo ciclo). Orientaciones didácticas. Madrid: Centro de Publicaciones del MEC.

\section{REFERENCIAS LEGISLATIVAS}

Constitución Española, BOE n $^{\circ} 311,29 / 12 / 1978$. Disponible en: https://www.boe.es/buscar/ act.php?id=BOE-A-1978-31229

Ley 14/1970, de 4 de agosto, General de Educación y Financiamiento de la Reforma Educativa. BOE, n ${ }^{\circ} 1874,06 / 09 / 1970,12525-12546$. Disponible en: https://boe.es/buscar/doc. php?id=BOE-A-1970-852

Ley Orgánica 1/1990, de 3 de octubre, de Ordenación General del Sistema Educativo. BOE, $\mathrm{n}^{\circ}$ 238, 04/10/1990, 28927-28942. Disponible en: https:/www.boe.es/eli/es/lo/1990/10/03/1

Ley Orgánica 10/2002, de 23 de diciembre de Calidad de la Educación. BOE, n 307, 24/12/2002, 45188-45220. Disponible en: https://www.boe.es/eli/es/lo/2002/12/23/10

Ley Orgánica 2/2006, de 3 de mayo, de Educación. BOE, nº 106, 04/05/2005, 17158-17207 Disponible en: https://www.boe.es/boe/dias/2006/05/04/pdfs/A17158-17207.pdf

Ley Orgánica 8/2013, de 9 de diciembre, para la mejora de la calidad educativa. BOE, $\mathrm{n}^{\circ} 295$, 10/12/2013. Disponible en: https://www.boe.es/eli/es/lo/2013/12/09/8/con

Orden de 25 de junio de 1974, que establece nuevos contenidos en las orientaciones pedagógicas del área social en la Segunda Etapa de Educación General Básica. BOE, $\mathrm{n}^{\circ}$ 162, 08/07/1974, 14185-14186. Disponible en: https://boe.es/boe/dias/1974/07/08/pdfs/ A14185-14186.pdf

Orden de 29 de noviembre de 1976 por la que se establecen nuevos contenidos en las orientaciones pedagógicas del área social en la segunda etapa de la Educación General Básica. BOE, $\mathrm{n}^{\mathrm{o}}$ 290, 03/12/1976, 24093-24094, Disponible en: https://boe.es/buscar/doc. php?id=BOE-A-1976-24474

Proyecto de Ley Orgánica por la que se modifica la Ley Orgánica 2/2006, de 3 de mayo, de Educación, 03/03/2020, Ministerio de Educación y Formación Profesional. Disponible en https://www.educacionyfp.gob.es/dam/jcr:8c7d3863-aaa7-4bbd-91b2-4d05bcb80286/ apl-lomloe-web2020-03-03.pdf

Real Decreto 1344/1991, de 6 de septiembre, por el que se establece el currículo de la Educación Primaria. BOE n $^{\circ}$ 220, 13/09/1991, 30226-30228. Disponible en: https:/www.boe.es/eli/ es/rd/1991/09/06/1344

Real Decreto 1318/2004, de 28 de mayo, por el que se modifica el Real Decreto 827/2003, de 27 de junio, por el que se establece el calendario de aplicación de la nueva ordenación del sistema educativo, establecida por la Ley Orgánica 10/2002, de 23 de diciembre, de 
Calidad de la Educación. BOE, n $130,29 / 05 / 2004,19924-19925$. Disponible en: https:// www.boe.es/eli/es/rd/2004/05/28/1318

Real Decreto 1513/2006, de 7 de diciembre, por el que se establecen las enseñanzas mínimas de la Educación primaria. BOE, no 293, 08/12/2006, Disponible en: https://www.boe.es/eli/ es/rd/2006/12/07/1513/con

Real Decreto 1631/2006, de 29 de diciembre, por el que se establecen las enseñanzas mínimas correspondientes a la Educación Secundaria Obligatoria. BOE, $\mathrm{n}^{\circ} 5,05 / 01 / 2007$, Disponible en: https://www.boe.es/eli/es/rd/2006/12/29/1631/con

Real Decreto 1190/2012, de 3 de agosto, por el que se modifican el Real Decreto 1513/2006, de 7 de diciembre, por el que se establecen las enseñanzas mínimas de la Educación Primaria, y el Real Decreto 1631/2006, de 29 de diciembre, por el que se establecen las enseñanzas mínimas correspondientes a la Educación Secundaria Obligatoria. BOE, $\mathrm{n}^{\circ} 186$, 04/08/2012, 55692-55704. Disponible en: https://www.boe.es/eli/es/rd/2012/08/03/1190

Real Decreto 126/2014, de 28 de febrero, por el que se establece el currículo básico de la Educación Primaria. BOE, no 52, 01/03/2014, Referencia: BOE-A-2014-2222. Disponible en: https://www.boe.es/buscar/pdf/2014/BOE-A-2014-2222-consolidado.pdf

Real Decreto 1105/2014, de 26 de diciembre, por el que se establece el currículo básico de la Educación Secundaria Obligatoria y del Bachillerato. BOE, $\mathrm{n}^{\circ} 3,03 / 01 / 2015,169-546$, Disponible en: https://www.boe.es/boe/dias/2015/01/03/pdfs/BOE-A-2015-37.pdf 